\title{
Non-existence and uniqueness results for fourth-order Hamiltonian systems
}

\author{
Mark A Peletier \\ Centrum voor Wiskunde en Informatica, 1090 GB Amsterdam, The Netherlands
}

Received 26 February 1999

Recommended by B Branner

Abstract. We give a classification of all bounded solutions of the equation

$$
u^{\prime \prime \prime \prime}+p u^{\prime \prime}+F^{\prime}(u)=0, \quad-\infty<t<\infty .
$$

in which $F$ is a general quartic polynomial and $p$ is restricted to various subsets of $(-\infty, 0]$. These results are obtained by combining an a priori estimate with geometric arguments in the $\left(u, u^{\prime \prime}\right)$-plane.

AMS classification scheme numbers: 58F05, 34A26, 34A34, 34A46

\section{Introduction}

Starting with the work of Toland [1] and Hofer and Toland [2], a technique has been developed to exploit the special structure of certain Hamiltonian systems with an indefinite kinetic energy [3-5]. This technique provides a geometrical tool akin to the phase plane analysis that has proven so powerful in the case of two-dimensional dynamical systems. Geometrical properties of a pseudo-phase space of reduced dimension are used to prove existence, uniqueness and many qualitative properties of solutions. The technique and the monotonicity property on which it is based are explained briefly in section 4 .

In this paper we combine this technique with an a priori estimate to study the solution set of the equation

$$
u^{\prime \prime \prime \prime}+p u^{\prime \prime}+F^{\prime}(u)=0, \quad-\infty<t<\infty, \quad p \leqslant 0 .
$$

While the methods used in this paper fall within the pseudo-phase plane setting mentioned above, equation (1) has also been studied using several other techniques, such as variational methods [6-9], and a topological shooting method [10-14]. The novelty that this paper brings to the pseudo-phase plane concept is the extension of the existing uniqueness and non-existence results, which typically are proven under certain restrictions on the solutions, to the set of all bounded solutions. A particularly interesting application is the change in bifurcation diagram that is discussed in section 2.

In equation (1) we are interested in potentials $F$ that have one or two wells, and for simplicity we assume that $F$ is an exact quartic. This is not essential, and for any double-well potential of similar type analogous results should hold. By exploiting the various symmetries present in (1) we can reduce the case of a general quartic to

$$
F(u)=a+\frac{1}{2} u^{2}-\frac{1}{3} u^{3}+\frac{1}{4} b u^{4}, \quad b>0,
$$


without losing generality. Note that one well is at $u=0$, that $F^{\prime \prime}(0)=1$, and that the second well, if it exists (which is the case for $0<b<\frac{1}{4}$ ) has $u>0$. When $b=\frac{2}{9}$, equation (1) is the extended Fisher-Kolmogorov equation with wells of equal depth (at $u=0$ and 3 ).

For a solution of (1), the Hamiltonian,

$$
H(u)=u^{\prime} u^{\prime \prime \prime}-\frac{1}{2} u^{\prime \prime 2}+\frac{1}{2} p u^{\prime 2}+F(u),
$$

is constant in time. By incorporating this constant into the definition of $F$ (in the zero-order term $a$ ) we can restrict ourselves to solutions with zero Hamiltonian, again without any loss of generality. In order to prevent needless repetition, we state here that all solutions are assumed to satisfy $H=0$.

Equation (1) has a constant solution $u \equiv \alpha$ if and only if $F^{\prime}(\alpha)=0$. If $b \geqslant \frac{1}{4}$, then $F$ has only one well, and the only bounded solutions are constants.

Theorem 1 (Single-well case). If $b \geqslant \frac{1}{4}$ and $p \leqslant 0$, then equation (1) has no bounded solutions other than (possibly) the unique constant solution.

Note that for $b=\frac{1}{4}$ the function $F$ has two stationary points: $F^{\prime}(0)=F^{\prime}(2)=0$, and therefore there are two stationary solutions. However, since $F(0) \neq F(2)$, these occur for different values of $a$, so that the constant solutions are indeed unique (as stated in theorem 1) under the condition $H=0$.

More interesting are the double-well potentials. In figure 1 we depict eight different classes of functions $F$, where the distinction is made on the basis of the distribution of signs and zeros of $F$, and their position with respect to the wells. When one well is at level zero, as in cases (b) and (f), we assume for definiteness that this is the well at $u=0$.

In the theorem below, the main result of this paper, we state uniqueness and non-existence results for bounded solutions. These hold for values of $p$ in intervals of the type $(-\infty, \bar{p}]$

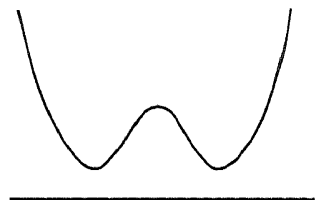

(a)

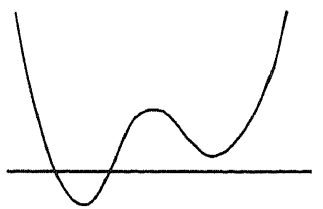

(d)

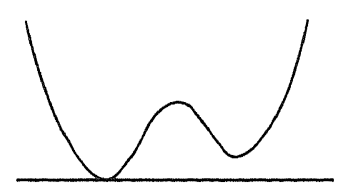

(b)

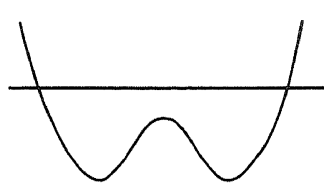

(e)

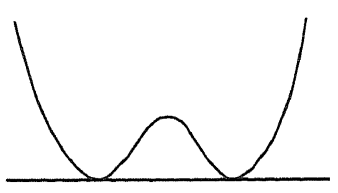

(c)

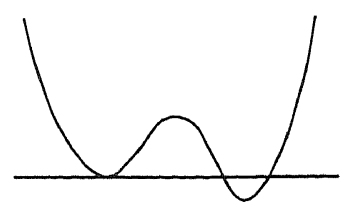

(f)

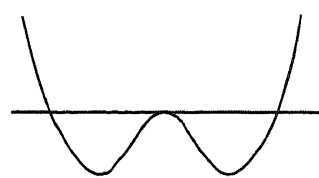

(g)

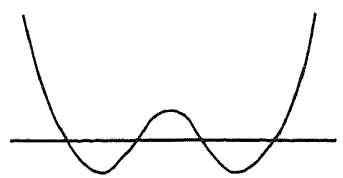

(h)

Figure 1. Eight different cases for $F$, classified by the character of the roots: (a) no zeros; (b) one double zero; (c) two double zeros; (d) two single zeros, case 1; (e) two single zeros, case 2; (f) two single, one double, case $1 ;(\mathrm{g})$ two single, one double, case 2 ; (h) four single zeros. 
or $(-\infty, \bar{p})$, where $-\infty<\bar{p} \leqslant 0$ is determined by the function $F$. It is of course possible to specify these limits exactly in terms of the values of $a$ and $b$, but here we only do that in isolated cases.

Theorem 2 (Double-well case). (i) In cases (a) and (d) there are no bounded solutions for any $p \leqslant p_{1}, p_{1} \leqslant 0$.

(ii) In case (b) the only bounded solution for $p \leqslant p_{2}, p_{2}>-2$, is the stationary solution $u \equiv 0$.

(iii) In case (c) the only bounded solutions for $p \leqslant-2$ are the constants $u \equiv 0$ and 3 and two heteroclinic solutions connecting 0 and 3 .

(iv) In case (e) there are no bounded solutions for any $p \leqslant 0$.

(v) In case $(f)$ the only bounded solutions for $p \leqslant p_{3}, p_{3} \leqslant-2$, are the stationary solution $u \equiv 0$ and a unique homoclinic orbit (homoclinic to zero).

(vi) In case ( $g$ ) the only bounded solution for $p \leqslant 0$ is the unique stationary solution.

(vii) In case ( $h$ ) the only bounded solution for $p \leqslant p_{4}, p_{4} \leqslant-2$, is a unique periodic orbit.

Remark 1. Some of the bounds on $p$ given above are sharp. For $p>-2$ (but not too large) in case (f) there exists a large collection of homoclinic orbits [4, 5]. In case (c), corresponding to the extended Fisher-Kolmogorov equation with equal wells, there are many heteroclinic connections for $p>-2$ [8-10], as well as periodic solutions [12] and non-periodic bounded solutions [11]. In case (b) homoclinic orbits have been found numerically (see $[15,16])$ for values of $p$ larger than -2 .

Remark 2. The fact that $F$ is assumed to be an exact quartic is not essential to most of the proof; however, it simplifies many of the arguments by creating a phase plane of simple geometry. For instance, the fact that regions $C$ and $C_{ \pm}$are strictly convex is used a number of times. As another example, the fact that $p_{2}>-2$ is the result of an explicit calculation for this function; for a different nonlinearity the result may also be different. In section 8 we discuss a generalization of these results to a different potential function.

Theorem 1 is proved in section 3 ; the subsequent sections discuss the proof of theorem 2 .

\section{Example: homoclinic orbits for a restiffening strut}

In the context of structural mechanics, equation (1) arises as a model for a thin elastic strut on a nonlinear elastic foundation. Such struts are known experimentally to buckle in a localized fashion when loaded axially (in this model the parameter $p$ represents the axial load). These localized profiles correspond to solutions of (1) that converge to zero at plus and minus infinity, i.e. homoclinic orbits. An interesting class of solutions of (1) is found as minimizers of the elastic strain energy

$$
\frac{1}{2} \int u^{\prime 2}+\int F(u)
$$

under the condition of fixed total shortening of the strut,

$$
\frac{1}{2} \int u^{\prime 2}=\lambda \text {. }
$$

For a description of the derivation of this model, see [17-19]. 
The function $F^{\prime}$ models the stress-strain relationship of the foundation, where $F^{\prime}(u)$ is the stress associated with a strain $u$. The localization property, which corresponds to the existence of homoclinic solutions of this minimization problem, is related to the decrease of $F^{\prime \prime}$ as $u$ increases from zero: the foundation loses stiffness as $u$ increases. It is relatively easy to verify that with a nonlinearity $F$ such that $F^{\prime \prime}$ increases as $|u|$ moves away from zero there are no homoclinic energy minimizers $\dagger$.

Recently, interest has turned to foundations that have a destiffening character for small $u$, but restiffen as $u$ becomes large. The function $F$ with $b>0$ (cases (f), (c) and (b)) is an example of such a foundation. The character of the homoclinic solutions is fundamentally different in the cases $0 \leqslant b<\frac{2}{9}$ (case (f)) and $b>\frac{2}{9}$ (case (b)). To illustrate this, consider the branch of primary solutions that bifurcates from $p=2$ and continues for decreasing values of $p$ (figure 2 ). For $0 \leqslant b<\frac{2}{9}$ this branch continues past $p=-2$ to $p=-\infty$, being the unique branch of solutions for $p \leqslant-2$ by theorem 2, case (f). (Not all of the features of the behaviour of the branch for $0 \leqslant p<2$ have been proven rigorously, but there is ample numerical and asymptotic evidence to support the conjectures made above.) As $b$ passes $\frac{2}{9}$, and the right-hand well crosses the zero axis, the branch of solutions for $p \leqslant-2$ disappears through a widening of the profile of the solutions; numerical evidence suggests that the profile converges to two heteroclinic orbits, connected in a multi-bump fashion, and the techniques of this paper could be used to prove this conjecture.

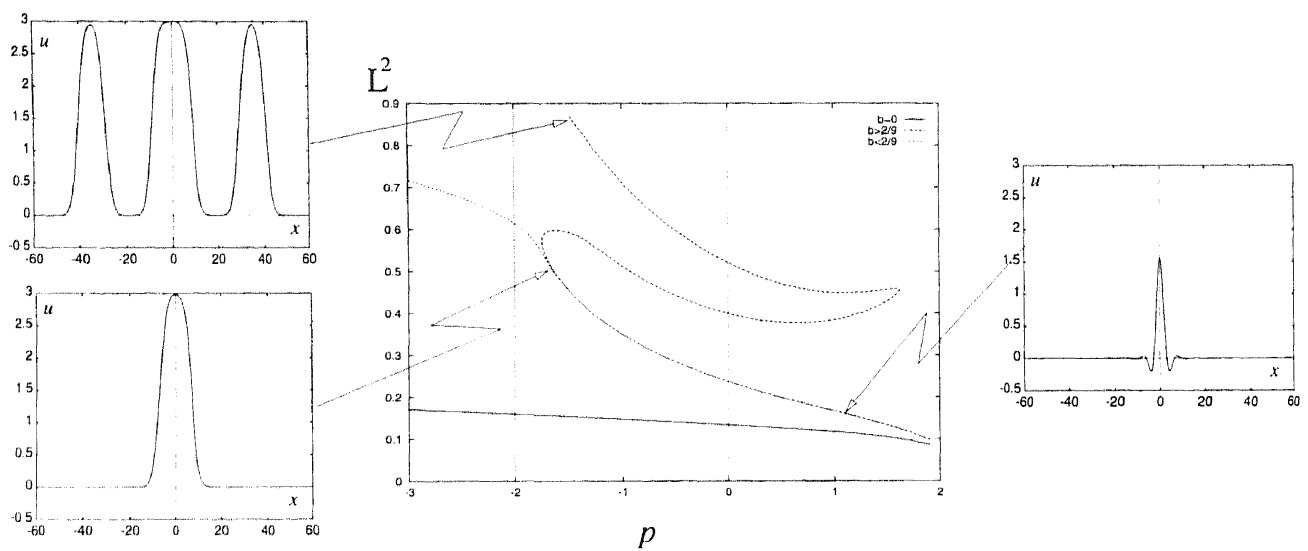

Figure 2. As $b$ passes $\frac{2}{19}$, the curve bifurcating from $p=2$ changes character (figure taken from |16|).

At the same time, the branch with $-2<p<2$ disconnects at $p=-2$ and performs an oscillatory movement, as shown in figure 2 . The results of theorem 2 for this situation can best be viewed in figure 3. For a detailed discussion of this transition, as well as other examples of systems in which such phenomena occur, see [15, 16].

$\dagger$ This can be verified by taking a sequence of functions of the form $u_{n}(t)=A_{n} \eta(t / n) \sin t$, where $\eta$ is a fixed cut-off function (e.g. $\eta(x)=\exp \left(-x^{2}\right)$ ) and $A_{n}$ is chosen to satisfy the constraint (3). For such a sequence the strain energy converges to $2 \lambda$, while by Fourier analysis it can be shown that for every non-zero $u \in H^{2}(\mathbb{R})$,

$$
\frac{1}{2} \int u^{\prime \prime 2}+\frac{1}{2} \int u^{2}>\int u^{\prime 2}
$$

Since $F^{\prime \prime} \geqslant 1$ and therefore $F(u) \geqslant u^{2} / 2$, this implies that the sequence $u_{n}$ converges to a strain energy level which is lower than that of every admissible fixed profile $u$. 


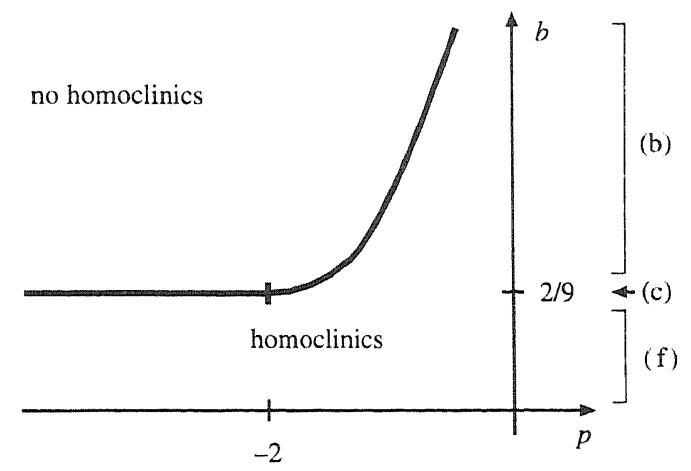

Figure 3. Graphical interpretation of (part of) theorem 2.

\section{Preliminary lemmas and $a$ priori bounds}

The following simple lemma has been proved many times; see for instance [20] or [13].

Lemma 3. Let $u$ be a solution of (1). If $u$ is monotonic and bounded for $t \geqslant 0$, then the constant $u_{\infty}=\lim _{t \rightarrow \infty} u(t)$ is a solution of $(1)$.

An important tool in this paper is the following a priori bound, which appears in [13] and generalizes a result from [11]. Define $I$ as the smallest closed interval such that the equation

$$
F(u)=s
$$

has exactly two solutions for all $s \in F(\mathbb{R} \backslash I)$ (see figure 4).

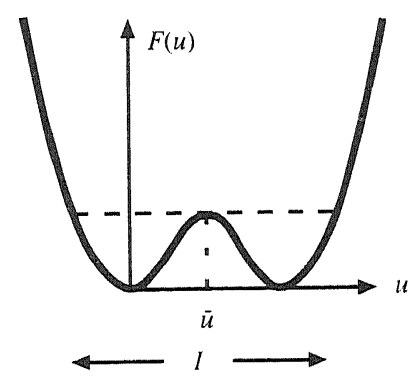

Figure 4. The function $F$ and the interval $l$.

Lemma 4. Let $p \leqslant 0$ and let $u$ be a non-constant solution of $(1)$ satisfying $H(u)=0$. If $u$ is bounded on $\mathbb{R}$, then $u(\mathbb{R}) \subset l$.

The proof of theorem 1 is a direct consequence of lemma 4 , since for a one-well potential $F$ we have $I=\{0\}$.

In the double-well case, there is a unique $\bar{u}$ at which $F$ has a local maximum. A direct consequence of theorem 1 is

Corollary 5. Let $u$ be a non-constant solution and set $R=u(\mathbb{R})$. Then $R$ contains $a$ neighbourhood of $\bar{u}$. 
If this were not the case, one could change the function $F$ outside of $R$ and create a single-well potential; thus the existence of the solution $u$ would contradict theorem 1 .

By this corollary, if $u$ is a non-constant solution, then either

(i) $u$ is monotonic; this implies the existence of two equilibria, and that $u$ is a heteroclinic orbit connecting the two; or

(ii) $u$ has local maxima above $\bar{u}$ and/or local minima below $\bar{u}$. These we call 'high' maxima and 'low' minima, and will serve in the sections below.

Case (e) in theorem 2 follows readily from this remark. There are no equilibria; therefore if $u$ is a solution, then it has a high local maximum. Let this maximum be attained at $t=0$; from (2) we have $F(u(0))=u^{\prime \prime 2} / 2 \geqslant 0$. By the form of $F$ this implies that $u(0) \notin I$, a contradiction.

\section{Case (c)}

Besides proving theorem 2 for case (c), we give in this section a brief description of the monotonicity property enjoyed by solutions of (1). For the uniqueness and non-existence results that we prove, only a small subset of the available techniques is necessary; the reader can find examples of complete treatments within this framework in [2-4].

We draw the $\left(u, u^{\prime \prime}\right)$-plane for case (c) (figure 5). Although this is a two-dimensional projection of the four-dimensional state space, we will call it the 'phase plane' because of the similarity of the methods employed here to phase plane analysis for two-dimensional autonomous ODEs. From the point of view of Hamiltonian systems one might also describe this plane as the 'configuration space', i.e. the space of the generalized positional (as opposed to moment) coordinates. Note that in case (c), where $b=\frac{2}{9}$, the function $F$ and equation (1) are invariant under the reflection $u \mapsto 3-u$.

The choice of the variables $u$ and $u^{\prime \prime}$ stems from the Hamiltonian condition $H=0$, which can be written as

$$
u^{\prime}\left(u^{\prime \prime \prime}+\frac{1}{2} p u^{\prime}\right)=V\left(u, u^{\prime \prime}\right):=\frac{1}{2} u^{\prime \prime 2}-F(u) .
$$

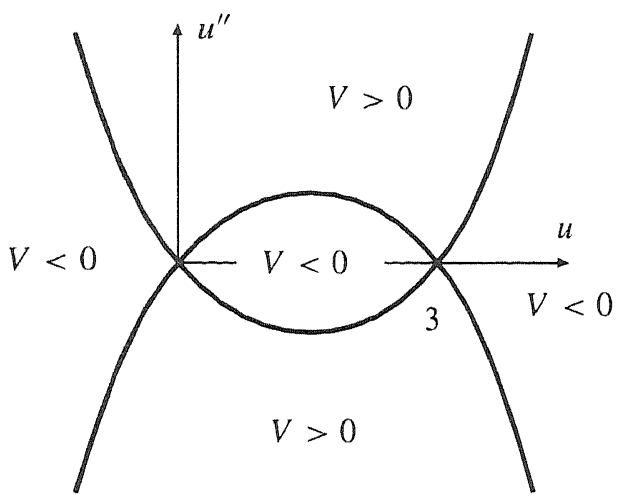

(a)

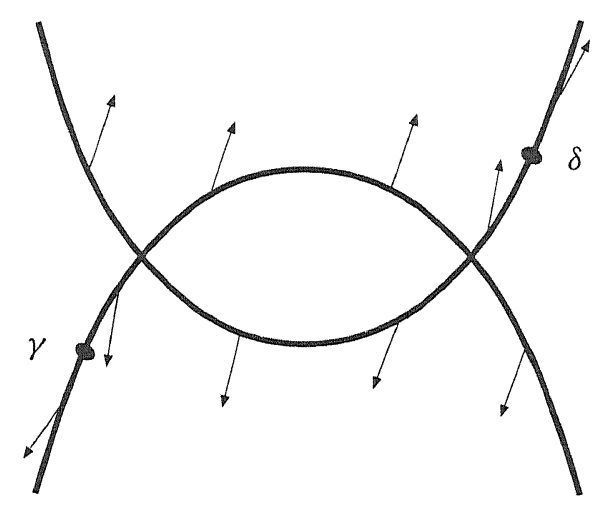

(b)

Figure 5. (a) The phase plane $u, u^{\prime \prime}$, with the set $\{V=0\} ;(b)$ an orbit with zero velocity leaves the set $\{V=0\}$ in the direction indicated by the arrows (drawn here for $p<-2$ ). 
If $V=0$, then either $u^{\prime}=0$ or $u^{\prime \prime \prime}=-p u^{\prime} / 2$. Therefore, if an orbit intersects the set $\{V=0\}$ at $t=t_{0}$, there are two possibilities: either

(i) the velocity $\left(u^{\prime}, u^{\prime \prime \prime}\right)$ is non-zero, and the direction of the orbit (given by the ratio $u^{\prime \prime \prime} / u^{\prime}$ ) is either $\infty$ (vertical) or $-p / 2$; or,

(ii) $u^{\prime}=u^{\prime \prime \prime}=0$, and $u$ is symmetric about $t_{0}$. This point is then called a turning point. In the phase plane the orbit returns along the same way as it arrived.

If an orbit is at a turning point on $\{V=0\}$, then the direction of the orbit (both in positive and in negative time, since the two orbits coincide) is fixed by the equation. Figure $5(b)$ shows these directions as arrows. We call 'magic points', marked with $\gamma$ and $\delta$, the points where the direction of the arrows changes side.

The sign of $V$ also implies a form of monotonicity. If $V<0$, then by equation (4) the velocity $\left(u^{\prime}, u^{\prime \prime \prime}\right)$ lies in a double cone as indicated by the shaded area below.
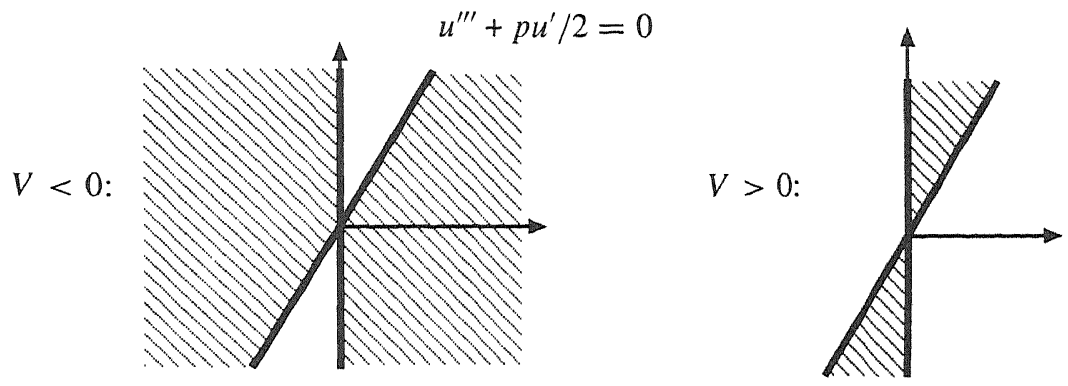

As long as $V$ remains strictly positive, the velocity cannot change from one cone to the other. Consequently, if $D$ is a bounded component of the set $\{V<0\}$, then an orbit with $\left(u, u^{\prime \prime}\right) \in D$ at $t=t_{0}$ must converge to $\partial D$ in both positive and negative time. Similar statements hold for the set $\{V>0\}$.

To prove theorem 2 for case (c), consider a non-constant solution $u$, and let $u$ attain a high maximum at $t=0$, so that $u(0) \geqslant \bar{u}, u^{\prime}(0)=0$, and $u^{\prime \prime}(0) \leqslant 0$. This implies that the orbit is at a point on the curve $\Gamma$ in figure 6 . The shaded vertical line on the left of this figure is the left boundary of $I$; a bounded solution cannot pass this line. If the orbit has zero velocity on $\Gamma$, then it enters the set $A$ (see the arrows in figure $5(b)$ ); if it has non-zero velocity, then it enters $A$ either in positive or in negative time. In both cases the monotonicity cone property implies that the orbit remains in $A$ and, since it is bounded, eventually leaves $I$. This is a contradiction.

Note that the hypothesis $p \leqslant-2$ is essential to this argument: as $p$ crosses the value -2 , the 'magic point' marked $\delta$ in figure $5(b)$ crosses the intersection at $u=3$. Therefore orbits with zero velocity on the curve between $\delta$ and the intersection point enter the inner region $\{V<0\}$ instead of $A$. Here the argument given above breaks down (and, in fact, theorem 2 does not hold for this case $[11,12])$.

The argument above proves that an orbit has no high maximum, and similarly by the symmetry $F(u)=F(3-u)$ we conclude that it has no low minimum either. This implies that it is monotonic, and therefore a heteroclinic orbit connecting the two equilibria, 0 and 3 . For the uniqueness of this heteroclinic orbit we refer to [13].

This concludes the proof of case (c) of theorem 2 . 


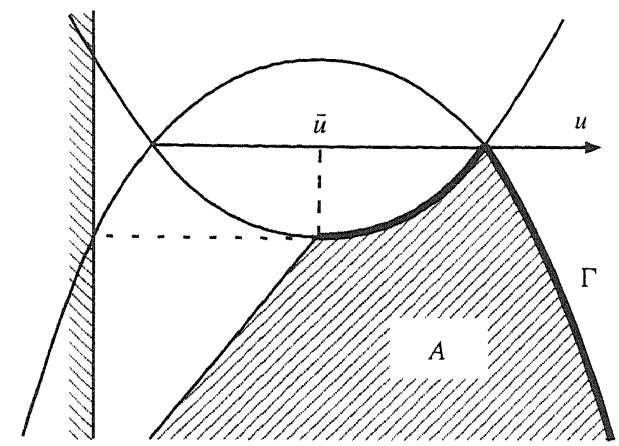

Figure 6. The left boundary of $A$ is given by the direction of the cone $\left(u^{\prime \prime \prime} / u^{\prime}=-p / 2\right)$. The direction of this cone implies that all bounded orbits passing through $\Gamma$ leave $I$.

\section{Cases (b) and (f)}

For cases (b) and (f) we shall prove theorem 2 in several steps. The first consists of the following lemma; other parts of the proof for these cases follow in sections 6 and 7 .

Lemma 6. In cases $(b)$ and $(f)$ for $p \leqslant-2$, a bounded non-constant solution has no local minima.

Proof. Lemma 6 uses the same idea as the previous section; both cases (b) and (f) are similar to case (c) in the left half of the phase plane (figure 7). We will discuss the proof only for (b), since the argument is nearly identical for $(\mathrm{f})$.

Suppose that a non-constant solution $u$ attains a low local minimum at $t=0$. Following the argument used in the previous section we conclude that the orbit intersects the line marked by $\Gamma$ in figure 8 , and enters the set $A$ (if necessary after inverting time). The boundedness of the orbit implies that it leaves the set $I$, which is a contradiction.

\section{Cases (a), (b) and (d)}

The common aspect of these three cases lies in the right half of the phase plane (see figure 9).

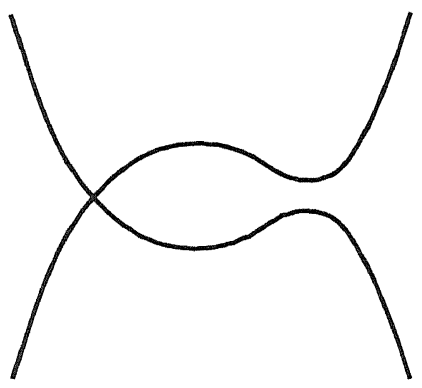

Case (b)

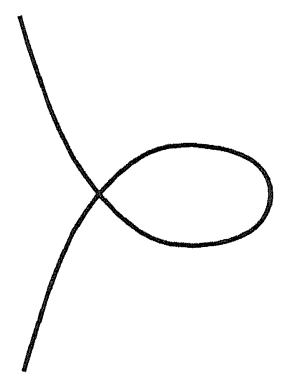

Case (f)

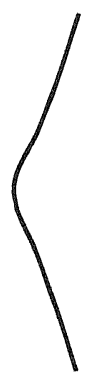

Figure 7. The phase planes in cases (b) and (f). 


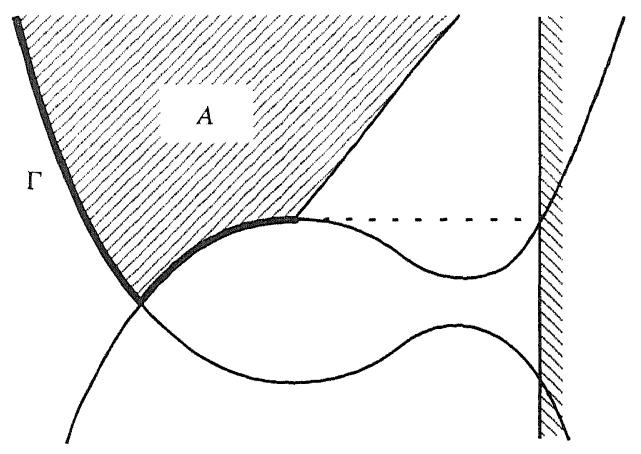

Figure 8 .

Lemma 7. In cases $(a)$, (b) and $(d)$ there exists $q_{1} \leqslant 0$, such that for $p \leqslant q_{1}$ a bounded non-constant solution has no high local maxima. In case (a) a bounded non-constant solution has no low local minima either.

Proof. We discuss case (b) first, and subsequently return to the other two cases.

Consider the curve $\Gamma$ in figure 10 . If this curve is considered as the graph of a function, $u^{\prime \prime}=g(u)$, then the derivative $g^{\prime}$ is bounded from above by a constant (say) $M$. Setting $q_{1}=-2 M$, it follows that for $p<q_{1}$ the tangent to $\Gamma$ nowhere has the direction $-p / 2$, and therefore there are no magic points (see section 4 ) on $\Gamma$. This implies that orbits starting with zero velocity on $\Gamma$ enter the set $A$.

If $p=q_{1}$, then there is exactly one point on $\Gamma$ where the cone and $\Gamma$ are tangent. By a continuity argument it follows that orbits starting from that point also enter $A$.

Repeating the argument from previous lemmas, we find that the existence of a high local maximum implies that the orbit enters $A$ from $\Gamma$, in positive or negative time, which in turn leads to a contradiction. This proves the lemma for case (b); the argument for case (d) is similar.

In case (a) this argument can be applied twice, both to a high maximum and to a low minimum. In general the ensuing values of $q_{1}$ will be different.

In these three cases we can now conclude the proof of theorem 2. For case (a), we note that there are no equilibria, and that therefore any bounded solution has high maxima and local

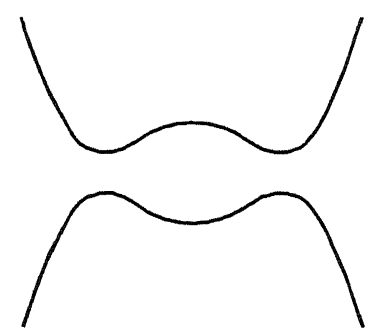

Case (a)

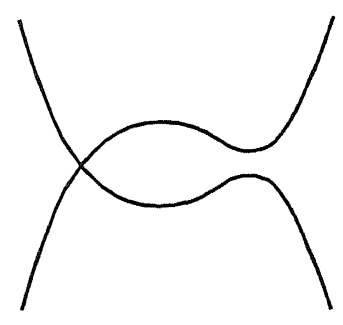

Case (b)
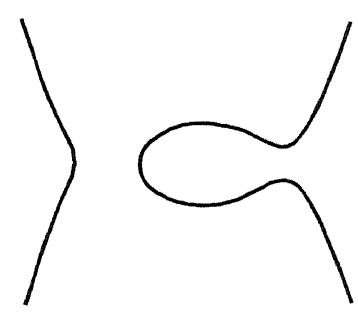

Case (d)

Figure 9. The phase planes in cases (a), (b) and (d). 


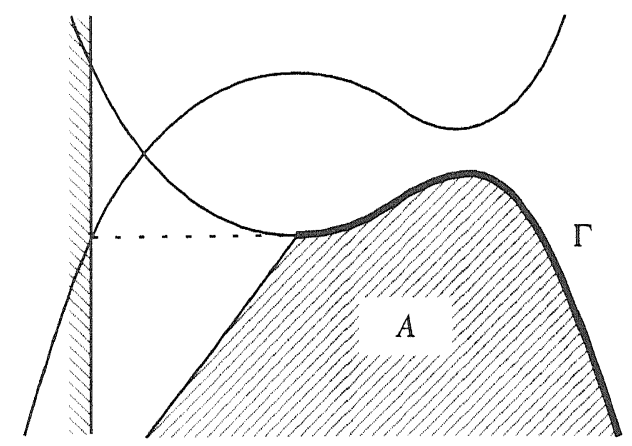

Figure 10.

minima. This contradicts lemma 7. For case (d), since there are no equilibria, there must be a high local maximum; by lemma 7 this contradicts the boundedness of the solution.

For case (b), lemmas 6 and 7 give the non-existence of non-constant solutions, for values of $p$ in the combined range $(-\infty,-2] \cup\left(-\infty, q_{1}\right)$. To complete the statement of theorem 2 we need to show that $q_{1}>-2$.

Lemma 8. For all $\frac{1}{4}>b>\frac{2}{9}$ we have $q_{1}>-2$.

Proof. As in the proof of lemma 7 we let $M$ denote the maximum of $g^{\prime}$ on the curve $\Gamma$; we need to prove that $M<1$ for all $b>\frac{2}{9}$.

Let $u_{1}=(1+\sqrt{1-4 b}) / 2 b$ be the location of the second well. Since $g$ is decreasing for $u>u_{1}$, we only need to concentrate on $\bar{u}<u<u_{1}$. Note that $F^{\prime \prime}\left(u_{1}\right)=u_{1}-2<1$. We write $F$ in the form

$$
F(u)-F\left(u_{1}\right)=\frac{1}{2} F^{\prime \prime}\left(u_{1}\right)\left(u-u_{1}\right)^{2}+h(u),
$$

where

$$
h(u)=\frac{1}{6} F^{\prime \prime \prime}\left(u_{1}\right)\left(u-u_{1}\right)^{3}+\frac{1}{24} F^{\prime \prime \prime \prime}\left(u_{1}\right)\left(u-u_{1}\right)^{4} .
$$

Note that $h(u)<0$ and $h^{\prime \prime}(u)<0$ for $\bar{u}<u<u_{1}$. We also define

$$
\hat{F}(u)=F\left(u_{1}\right)+\frac{1}{2} F^{\prime \prime}\left(u_{1}\right)\left(u-u_{1}\right)^{2}=F(u)-h(u) .
$$

Let $g^{\prime}(u)$ be maximal in $u=\tilde{u}$. There is a uniquely defined $\hat{u}$ such that $\hat{F}^{\prime}(\hat{u})=F^{\prime}(\tilde{u})$; since $h^{\prime \prime}<0$ it follows that $u_{1}>\hat{u}>\tilde{u}$, and it is straightforward to show that $\hat{F}(\hat{u})<F(\tilde{u})$. We then have

$$
g^{\prime}(\tilde{u})=\frac{F^{\prime}(\tilde{u})}{\sqrt{2 F(\tilde{u})}}<\frac{\hat{F}^{\prime}(\hat{u})}{\sqrt{2 \hat{F}(\hat{u})}}=\frac{F^{\prime \prime}\left(u_{1}\right)\left(\hat{u}-u_{1}\right)}{\sqrt{2 F\left(u_{1}\right)+F^{\prime \prime}\left(u_{1}\right)\left(\hat{u}-u_{1}\right)^{2}}}<\sqrt{F^{\prime \prime}\left(u_{1}\right)} .
$$

Since $F^{\prime \prime}\left(u_{1}\right)<1$ for $\frac{2}{9}<b<1$, the result is proved. 


\section{Cases (f) and (h)}

The uniting characteristic of these two cases is again a half of the phase plane (figure 11).

We define the closed convex set $C$ as in the figures. The work of Hofer and Toland [2], as well as many others, shows the existence of an even homoclinic orbit for $p \leqslant-2$ in case ( $f$ ), and of a periodic orbit for $p \leqslant 0$ in case (h). Both do not leave the set $C$. Note that the set $C$ is strictly convex for this function $F$.

In [5] it was proved for $b=0$ that any homoclinic orbit with $p \leqslant-2$ is even. Here we generalize this result to $b>0$, and to more general bounded orbits. The result in [5] is based on a monotonicity argument that requires the function $F^{\prime}(u)-u$ to be non-increasing. For $b=0$ this is the case for all $u \geqslant 0$; for positive $b, u$ is restricted to the set $W=[0,2 / 3 b]$.

Lemma 9. Let $p \leqslant-2$ and let $u$ be a bounded solution of (I) such that the range of $u$ is a subset of $W$. Then $u$ is even about any maximum or minimum.

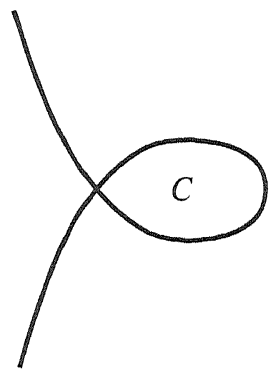

Case (f)
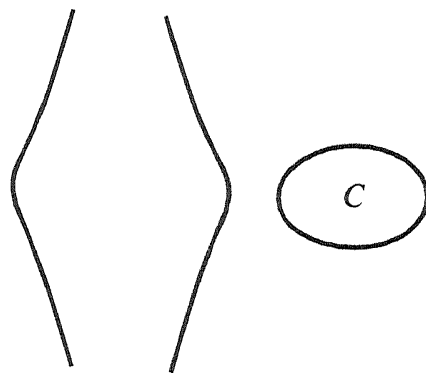

Case (h)

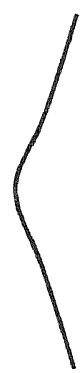

Figure 11. The phase planes in cases (f) and (h).

Proof. Let $\lambda \in(0,1]$ satisfy $\lambda+\lambda^{-1}=-p$. Let $u$ have a maximum at $t=0$. If $u^{\prime \prime \prime}(0)=0$, then $u$ is even about zero, and the proof is complete. Suppose, to force a contradiction, that $u^{\prime \prime \prime}(0)>0$ (the case $u^{\prime \prime \prime}(0)<0$ follows by reflection). Set $v(x)=u(-x)$; then we have

$$
\begin{array}{ll}
u(0)=v(0), & u^{\prime}(0)=v^{\prime}(0), \\
\left(u^{\prime \prime}-\lambda u\right)(0)=\left(v^{\prime \prime}-\lambda v\right)(0), & \left(u^{\prime \prime \prime}-\lambda u^{\prime}\right)(0)>\left(v^{\prime \prime \prime}-\lambda v^{\prime}\right)(0) .
\end{array}
$$

There exists a $\sigma>0$ such that $z=u-v>0$ on $(0, \sigma)$. Set $w=u^{\prime \prime}-v^{\prime \prime}-\lambda(u-v)$. We then have

$$
w(0)=0, \quad w^{\prime}(0)>0,
$$

and

$$
w^{\prime \prime}-\lambda^{-1} w=-\left(F^{\prime}(u)-u\right)+F^{\prime}(v)-v>0 \quad \text { on }(0, \sigma) .
$$

so that $w>0$ on $(0, \sigma)$. This implies that

$$
z^{\prime \prime}-\lambda z>0 \quad \text { on }(0, \sigma)
$$

so that $z(\sigma)>0$; consequently $z>0$ on $(0, \sigma]$, and by reasoning by contradiction we find that we can take $\sigma=\infty$. Going back to (5) we find that $z(t) \rightarrow \infty$ as $t \rightarrow \infty$, which contradicts the boundedness of $u$ and $v$.

We shall show, starting with the lemma below, that in fact no bounded solution can leave the set $C$. 


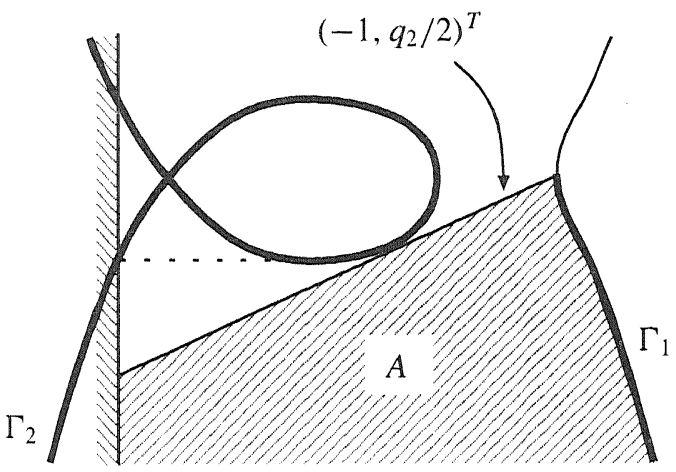

Figure 12.

Lemma 10. In cases $(f)$ and $(h)$ there exists $q_{2}<0$ such that for $p \leqslant q_{2}$ a bounded solution cannot have a high local maximum outside $C$. In case (h) the same holds for a low local minimum.

Proof. Consider the phase plane of case (f), in figure 12. Let $u$ have a high local maximum outside $C$, that therefore lies on the curve $\Gamma_{1}$. There exists a $q_{2}<0$ such that any half-line starting on $\Gamma_{1}$ and extending in the direction $\left(-1, q_{2} / 2\right)^{T}$ leaves $I$ before intersecting $\Gamma_{2}$. For $p \leqslant q_{2}$ the same is true for any orbit that enters $A$ from $\Gamma_{1}$. Using the same arguments as before, and noting that orbits with zero velocity that start on $\Gamma_{1}$ enter $A$, this implies a contradiction.

Lemma 11. In cases $(f)$ and $(h)$ for $p \leqslant-2$, if an orbit has a local maximum on $\partial C$, then the orbit is even about the maximum and lies within $C$ for all time. In case $(h)$ the same is true for a local minimum.

Proof. In case (f) the orbit has no low local minima by lemma 6 , so that $u(t) \rightarrow 0$ as $t \rightarrow \pm \infty$ and $u$ is monotonic whenever $u \leqslant \bar{u}$. Since $C \subset[0,3] \subset[0,2 / 3 b]$ we therefore have $u(\mathbb{R}) \subset W$ so that the orbit is even by lemma 9 .

We identify on $\partial C$ three (open) sections $\Gamma_{1}, \Gamma_{2}$ and $\Gamma_{3}$, and the two 'magic points' $\alpha$ and $\beta$ (figure 13(a)). We concentrate on the maximum of the orbit, which lies on $\Gamma_{2} \cup \Gamma_{3} \cup\{\beta\}$, and leaves $\partial C$ with zero velocity since the orbit is even. Note that an orbit starting in $\alpha$ has a local minimum and therefore is not a candidate.

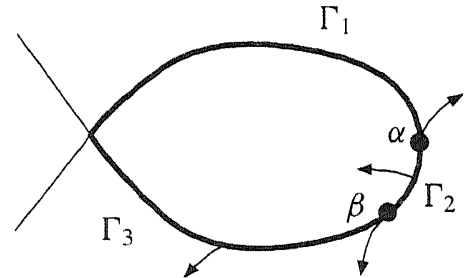

(a)

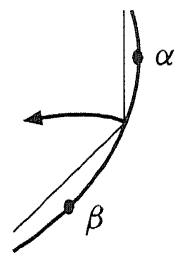

(b)

Figure 13. The arrows in $(a)$ indicate the direction of orbits that start with zero velocity on $\partial C$ The direction of orbits starting in $\alpha$ and $\beta$ follows from a continuity argument. 


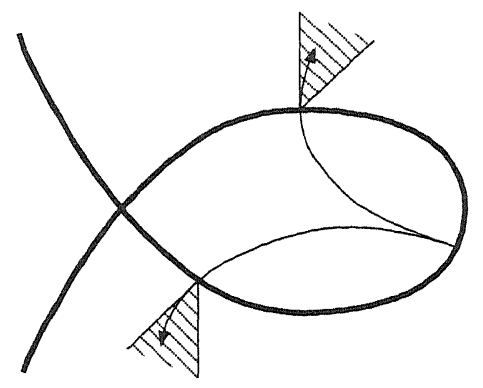

Figure 14.

If the orbit starts on $\Gamma_{2}$, then it remains inside the cone shown in figure 13(b) and can only exit $C$ through $\Gamma_{1}$ or $\Gamma_{3}$. Figure 14 shows how both of these alternatives lead to a nonmonotonic orbit; exit via $\Gamma_{1}$ yields a local minimum on $\Gamma_{1}$, and exit through $\Gamma_{3}$ implies that the orbit cannot tend to zero without $u$ taking negative values. Therefore this case leads to a contradiction.

If the orbit starts on $\Gamma_{3} \cup\{\beta\}$, then the non-monotonicity mentioned above again applies, leading to a contradiction.

A similar argument applies to case (h).

We now conclude the proof of theorem 2 for the remaining cases. For case (f), by lemmas 10 and 11 , for $p \leqslant \min \left\{q_{2},-2\right\}$ a bounded orbit is even, remains inside $C$, and is homoclinic to zero. The uniqueness for orbits of this type is proven in [3].

In case ( $h$ ) a bounded orbit is also even and remains inside $C$, and by slightly adapting the uniqueness proof of [3] these orbits can also be shown to be unique.

Case $(\mathrm{g})$ is a special example of case $(\mathrm{h})$, one in which the set $C$ is reduced to one point. This point is the unique stationary point, and by the uniqueness for case $(\mathrm{h})$ the unique bounded solution. This concludes the proof of theorem 2 .

\section{Generalizations}

As an example of a possible generalization of theorem 2 we consider here equation (1) with nonlinearity $F(u)=a+u^{2} / 2-u^{4} / 4+b u^{6} / 6$ (figure 15 ). This nonlinearity is interesting in the context of structural mechanics and structural geology (see section 2 and [16]) because of the symmetry around zero. In this application the emphasis is on solutions that are homoclinic or heteroclinic, so we only consider $a=0$. As before, the Hamiltonian is always assumed to be zero.

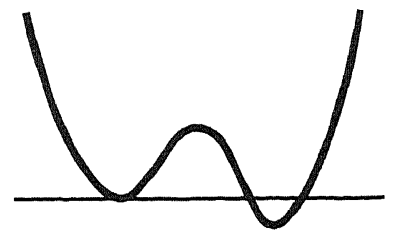

(a)

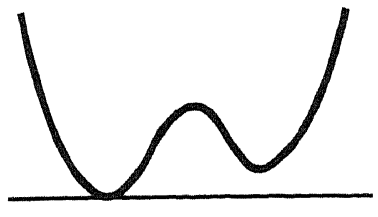

(b)

Figure 15. The nonlinearity $F(u)=a+u^{2} / 2-u^{4} / 4+b u^{6} / 6 .(a) 0<b<\frac{3}{16} ;(b) b>\frac{3}{16}$. 


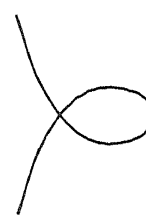

(a)

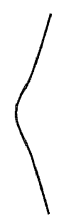

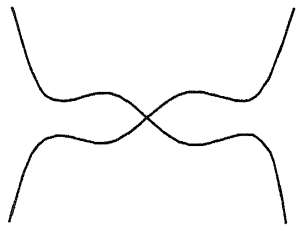

(b)

Figure 16. As $b$ passes the value $\frac{3}{16}$ the convex regions $C_{ \pm}$open up. $(a) 0<b<\frac{3}{16}$; $(b) b>\frac{3}{16}$.

Fixing $a=0$, the phase plane contains two convex regions $C_{ \pm}$for $0<b<\frac{3}{16}$ that open up as $b$ passes $\frac{3}{16}$ (figure 16). This is analogous to the procession of cases (f)-(c)-(b) of figure 1.

Applying the same ideas as above we find

Theorem 12. (i) When $0<b<\frac{3}{16}$, there are two solutions homoclinic to zero for any $p \leqslant-2 ;$ when $p \leqslant q_{1} \leqslant-2$, these are unique in the class of bounded solutions.

(ii) When $b=\frac{3}{16}$, there are two heteroclinic solutions for $p \leqslant-4$, one connecting -2 and 0 and one connecting 0 and 2 (and their mirror images under $t \mapsto-t$ ). The only other bounded solutions are the three equilibrium solutions.

(iii) When $b>\frac{3}{16}$ there is $a q_{2} \leqslant-2$ such that there are no bounded solutions for $p \leqslant p_{2}$.

Proof. The simplest case is $b>\frac{3}{16}$ (figure 17). Here the argument is very similar to that in section 6 for case (b): there is a number $q_{2} \leqslant 0$, given by the geometry of the curve $\Gamma$, such that if $p \leqslant q_{2}$ then the monotonicity cone points inwards from any point on $\Gamma$. The rest of the argument is identical. The final statement is that there are no bounded orbits for $p \leqslant q_{2}$. Note that in contrast to the case considered above we have $q_{2} \leqslant-2$ for all $b>\frac{3}{16}$, since the direction of $\Gamma$ at the origin is $( \pm 1, \pm 1)^{T}$. When $b=\frac{3}{16}$ the phase plane is a variation on case (c) discussed above (figure 18). Any non-monotonic bounded solution either has a low local minimum (i.e. below $\bar{u}$ ) or a high local maximum (i.e. above $-\bar{u}$ ). Concentrating on the high local maximum, and using the convexity of the regions $C_{ \pm}$, the monotonicity cone points into $A$ from any point on $\Gamma$, provided $p \leqslant-4$ (the value of -4 is related to the direction of $\Gamma$ at $( \pm 2,0)$ which is $( \pm 1, \pm 2)$ ). Consequently, the only bounded orbits are those that are monotonic; these connect two equilibria, and by $[2,3]$ these exist for the connections $-2 \leftrightarrow 0$ and $0 \leftrightarrow 2$. These are unique within the class of orbits that remain inside $C_{-}$or $C_{+}$. With similar arguments as above it follows that any bounded orbit must remain inside $C_{-} \cup C_{+}$.

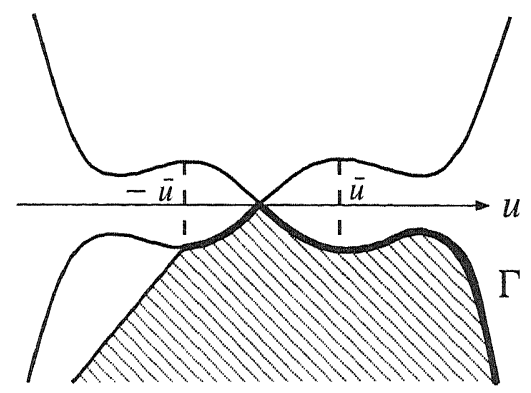

Figure 17. 


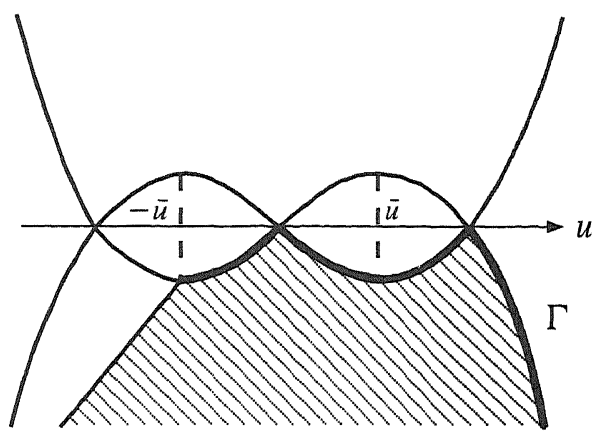

Figure 18.

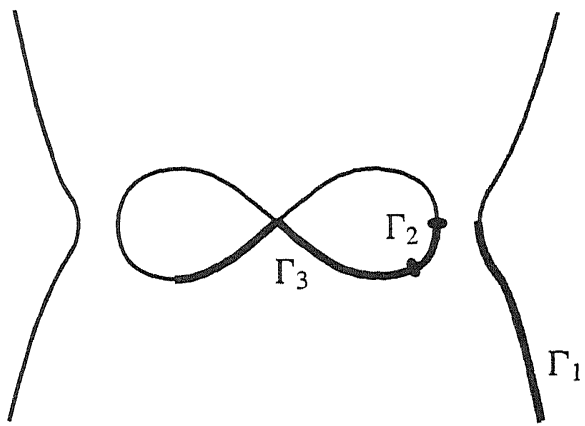

Figure 19.

There is a theoretical possibility of a third connection, between -2 and 2 , provided the orbit does not leave the set $C_{-} \cup C_{+}$; this is excluded for $p<-2$ by the imposed directions of the orbit at the origin (since the orbit has to pass the origin with non-zero velocity, the direction $u^{\prime \prime \prime} / u^{\prime}$ is either infinity or $-p / 2$; in both cases this is not possible for orbits remaining inside $\left.C_{-} \cup C_{+}\right)$.

For $0<b<\frac{3}{16}$ the results of $[2,3]$ give the existence of two homoclinic orbits for $p \leqslant-2$, again one in each of the regions $C_{ \pm}$, which are unique among solutions that remain inside $C_{ \pm}$. Lemma 10 and its proof hold unchanged for this situation, so that there is a $q_{1}<0$ such that for $p \leqslant q_{1}$ there are no bounded orbits with a maximum on $\Gamma_{1}$ (see figure 19). For orbits having a high local maximum on $\Gamma_{2}$ or $\Gamma_{3}$ the argument is similar to that of lemma 11: such orbits are monotonic by lemma 9 , and if an orbit leaves $C_{-} \cup C_{+}$then this monotonicity is violated.

Remark 3. When $b=\frac{3}{16}$, the case of all wells having equal depth, the value $p=-4$ marks the passing of magic points through the two outer equilibria. Therefore, using the techniques of [4] one expects the appearance for $p=-4^{+}$of a large number of solutions that are homoclinic to \pm 3 . Therefore the condition $p \leqslant-4$ is sharp for the uniqueness result.

\section{Acknowledgments}

The author wishes to thank Boris Buffoni, Alan Champneys and John Toland, for the many and fruitful discussions that led to this paper, as well as the Centre for Nonlinear Mechanics, Bath, 
for providing such a stimulating environment. In addition, gratitude is due to Jan Bouwe van den Berg for mentioning lemma 4. This work was partially supported by the EPSRC, through grant GR/L17177 of the Applied Nonlinear Mathematics Initiative.

\section{References}

11] Toland J F 1981 Solitary wave solutions for a model of the two-way propagation of water waves in a channel Math. Proc: Camb. Phil. Soc: $90343-60$

12) Hofer H and Toland J F 1984 On the existence of homoclinic, heteroclinic and periodic orbits for a class of indefinite Hamiltonian systems Math. Ann. $268387-403$

[3] Amick C J and Toland J F 1992 Homoclinic orbits in the dynamic phase-space analogy of an elastic strut Eur. J. Appl. Math. 397-114

14] Champneys A R and Toland J F 1993 Bifurcation of a plethora of multi-modal homoclinic orbits for autonomous Hamiltonian systems Nonlinearity 6 665-772

[5] Buffoni B, Champneys A R and Toland J F 1996 Bifurcation and coalescence of a plethora of homoclinic orbits for a Hamiltonian system J. Dynam. Stab. Syst. $8221-81$

[6] Buffoni B 1996 Periodic and homoclinic orbits for Lorentz-Lagrangian systems via variational methods Nonlin. Anal. 26 443-62

[7] Buffoni B and Séré E 1996 A global condition for quasi-random behaviour in a class of conservative systems Commun. Pure Appl. Math. 49 285-305

[8] Kalies W D and van der Vorst R C A M 1996 Multitransition homoclinic and heteroclinic solutions of the extended Fisher-Kolmogorov equation J. Differ. Eq. 131 209-28

19] Kalies W D, Kwapisz J and van der Vorst R C A M 1998 Homotopy classes for stable connections between Hamiltonian saddle-focus equilibria Commun. Math. Phys. 193 337-71

[10] Peletier L A and Troy W C 1995 Spatial patterns described by the extended Fisher-Kolnogorov (EFK) equation: kinks Differ. Integr. Eq. 8 1437-60

[11] Peletier L A and Troy W C 1996 Chaotic spatial patterns described by the extended Fisher-Kolmogorov equation J. Differ. Eq. $129458-508$

[12] Peletier L A and Troy W C 1997 Spatial patterns described by the extended Fisher-Kolnogorov (EFK) equation: periodic solutions SIAM J. Math. Anal. 28 1317-53

[13] van den Berg G J B 1997 Uniqueness of solutions for the extended Fisher-Kolmogorov equation Technical Report W 97-24 University of Leiden

[14] Peletier L A, Rotatiu-Bruma A I and Troy W C 1998 Pulse-like spatial patterns described by higher-order model equations J. Differ. Eq. 150 124-87

[15] Woods P D and Champneys A R 1999 Heteroclinic tangles and homoclinic snaking in the unfolding of a degenerate reversible Hamiltonian Hopf bifurcation Physica D 129 147-70

[16] Hunt G W, Champneys A R, Woods P D, Peletier M A, Ahmer Wadee M, Budd C J and Lord G L 1999 Cellular buckling in long structures, to appear

[17] Hunt G W, Bolt H M and Thompson J M T 1989 Evolution of localized folding for a thin elastic layer in a softening visco-elastic medium Pure Appl. Geophys. 146 229-52

[18] Whiting A I M and Hunt G W 1997 Evolution of nonperiodic forms in geological folds J. Math. Geol. 29 705-23

[19] Peletier M A Sequential buckling: a variational analysis, to appear

[20] Hadamard J 1896 Sur certaines proprietés des trajectoires en dynamique J. Math. Ser. $5331-387$ 